\title{
CONJUGACY CLASSES OF NON-CONNECTED SEMISIMPLE ALGEBRAIC GROUPS
}

\author{
S. MOHRDIECK \\ Mathematisches Institut \\ Universität Basel \\ Rheinsprung 21 \\ CH 4051 Basel, Switzerland \\ mohrdis@math.unibas.ch
}

\begin{abstract}
Consider a non-connected algebraic group $\widetilde{G}=G \ltimes \Gamma$ with semisimple identity component $G$ and a subgroup of its diagram automorphisms $\Gamma$. The identity component $G$ acts on a fixed exterior component $G \tau, i d \neq \tau \in \Gamma$ by conjugation. In this paper we will describe the conjugacy classes and the invariant theory of this action. Let $T$ be a $\tau$-stable maximal torus of $G$ and its Weyl group $\mathcal{W}$. Then the quotient space $G \tau / / G$ is isomorphic to $(T /(1-\tau)(T)) / \mathcal{W}^{\tau}$. Furthermore, exploiting the Jordan decomposition, the reduced fibres of this quotient map are naturally associated bundles over semisimple $G$-orbits. Similar to Steinberg's connected and simply connected case [22] and under additional assumptions on the fundamental group of $G$, a global section to this quotient map exists. The material presented here is a synopsis of the Ph.D thesis of the author, cf. [15].
\end{abstract}

\section{Introduction}

We consider non-connected algebraic groups $\widetilde{G}$, over an algebraically closed field $k$, having semisimple identity component $G$ with Dynkin diagram $\Delta$. The aim of this paper is to provide an understanding of the invariant theory and the classes of the conjugacy action of $G$ on $\widetilde{G}$.

The study of non-connected semisimple algebraic groups has, recently, attracted some attention in the mathematics and physics literature. For example, Digne and Michel [5] developed a Deligne-Lusztig theory for these groups. In particular, their conjugacy classes play an important role in physics due to their connection to so called $D$-branes in the Wess-Zumino-Witten model $[1,6,16]$.

But, there are also some older results. Gantmakher [7], see also [8], introduced an analogue of a maximal torus containing a given outer automorphism of a semisimple Lie algebra over $\mathbb{C}$; and Kostant [11] proved a generalisation of Weyl's character formula for non-connected compact Lie groups.

Recall that non-connected groups $\widetilde{G}$ appear naturally as centralisers of semisimple elements in non-simply connected, connected, semisimple algebraic groups. The nonconnected groups $\widetilde{G}$ we are considering in this paper are semi-direct products:

$$
\widetilde{G}=G \rtimes \Gamma,
$$

Received April 29, 2002. Accepted July 1, 2003. 
where $\Gamma$ is a subgroup of the group of diagram automorphisms Aut $\Delta$. Now, let us fix a nontrivial element $\tau \in \Gamma$. In this paper we investigate the invariant theory and orbit structure of the conjugacy action of $G$ on the exterior component $G \tau$ of $\widetilde{G}$ :

$$
G \times G \tau \rightarrow G \tau, \quad(g, h \tau) \mapsto g h \tau g^{-1} .
$$

This conjugacy action is well understood in the connected case (i.e., $\tau=$ id) due to the well-known results by Steinberg $[22,25]$. Furthermore, in the non-connected case, partial results in this direction have been obtained, before, by Spaltenstein [19] who dealt with the $A_{n}$-case by direct computation. Following the ideas of Steinberg's, $[22,25]$, we will provide a more thorough conceptual approach here. However, his methods require an adaptation to our more general situation. For example, we have to make use of an analogue of a maximal torus, called the Cartan subgroup. E.g. if $\Gamma^{\prime}<\Gamma$ is the subgroup generated by $\tau$ and $T_{0}^{\tau}$, the identity component of the $\tau$-fixed points on $T$, then the group $C:=T_{0}^{\tau} \times \Gamma^{\prime}<\widetilde{G}$ will be a Cartan subgroup. One of its key properties is that it has finite index in its normaliser $N_{\widetilde{G}}(C)$. The finite group $\widetilde{\mathcal{W}}:=N_{G}(C) / T_{0}^{\tau}$ turns out to be a semi-direct product $H \rtimes \mathcal{W}^{\tau}$ of the fixed point Weyl group and a finite abelian group $H$. We will also prove that the quotient $T_{0}^{\tau} \tau / H$ is isomorphic to the coinvariant torus $T /(1-\tau)(T)$.

For our discussion we impose the following restrictions on the field $k$ : First, $k$ should be algebraically closed. Its characteristic must not divide the order of $\tau$ and is required to be unequal to 2 .

The main result of this paper will be the following theorem:

Theorem 1.1. (i) The inclusion map $i: T_{0}^{\tau} \tau \rightarrow G \tau$ induces an isomorphism $\widetilde{\iota}$ of the quotient spaces by the commutative diagram

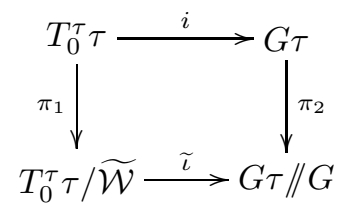

(ii) For simply-connected $G$ the quotient space is an affine space.

Let us add that for simply-connected $G$ the quotient map can, similar to Steinberg's case in [22], be described in terms of characters of $\widetilde{G}$.

Remark 1. In a recent paper, Joyner [10] proved the same result in characteristic 0 using a similar line of reasoning. However, his proof contains a minor gap, since he incorrectly identified $T_{0}^{\tau}$ and the coinvariant torus and hence overlooked the appearance of the extra part $H$ of the outer Weyl group. As our proof shows, this can easily be repaired.

Furthermore, we prove in Section 4 the existence of a section to the quotient map in this case.

Theorem 1.2. If $G$ is simply-connected, the quotient map $\pi: G \tau \rightarrow G \tau / / G$ admits a section $C$ whose image consists of a 'twisted Coxeter cell' $U_{c_{\tau}} c_{\tau} \subset G \tau$,

$$
C: T_{0}^{\tau} / \widetilde{\mathcal{W}} \rightarrow G \tau .
$$


Here $c_{\tau}$ is a twisted Coxeter element introduced by Steinberg (see, e.g., [20, Section 7]) and $U_{c_{\tau}}$ is a product of $s=\operatorname{dim} T_{0}^{\tau}$ unipotent one-parameter subgroups of the unipotent radical $U$ of $B$. Additional properties of $C$ imply reducedness and normality of the schematic fibres of $\pi$.

Steinberg's description of conjugacy classes was used by Slodowy in [18] (following Brieskorn [2]) to establish a connection between the theory of simple algebraic groups and that of simple singularities. Such a link can can be established here as well. However, there are no really new results and methods, and so we shall not deal with this here. See [15, Chapter 6] for details. Nonetheless, let us mention one aspect, a generalisation of Grothendieck's simultaneous resolution to our setting.

Theorem 1.3. Let $\operatorname{char}(k)>r k G+1$ or $\operatorname{char}(k)=0$ and let $\tau$ act without fixed points on the fundamental group of $G$. Then there exists a simultaneous resolution of singularities of the quotient map of $\pi: G \tau \rightarrow G \tau / / G$.

For the existence of the simultaneous resolution we need that $\operatorname{char}(k)$ does not divide the fundamental group of the centraliser $C_{G}(t \tau)$ for any $t \tau \in T_{0}^{\tau} \tau$. This is guaranteed by the above restriction.

This paper is organised as follows. In Section 2 we compile basic properties of the folding of root systems and discuss the lifting of exterior automorphisms to $G$.

The notion of a Cartan subgroup will be introduced in Section 3 and its basic properties will be discussed. Furthermore, we will prove Theorem 1.1.

Section 4 will deal with the existence of a cross section to the quotient map.

In Section 5 we state some further results in this setting.

\section{Preliminaries}

Here we assemble the basic concepts and notation used in this paper.

We will start with root systems and their foldings with respect to an outer automorphism. Let $R$ be a reduced root system with Dynkin diagram $\Delta, \mathcal{W}$ its Weyl group and $\Pi$ a set of simple roots. The real vector space generated by $R$ will be denoted by $V$. It is a well-known fact that the group of automorphisms Aut $(R)$ of $R$ is a semi-direct product $\operatorname{Aut}(R)=\operatorname{Aut}(\Delta) \ltimes \mathcal{W}$. Here Aut $(\Delta)$ is the group of diagram automorphisms. Recall that for irreducible $R$, the group Aut $(\Delta)$ is the symmetric group $S_{3}$ in the $\mathrm{D}_{4}$ case, $\mathbb{Z} / 2 \mathbb{Z}$ in the $\mathrm{A}_{n}, \mathrm{D}_{n}, n \geq 5$ and $\mathrm{E}_{6}$ while it is trivial in all other cases.

For a fixed $\tau \in \operatorname{Aut}(\Delta)$ we denote by $V^{\tau}$ the fixed point vector space. Define the projection map $p: V \rightarrow V^{\tau}$ by $v \mapsto p(v):=\frac{1}{\operatorname{ord} \tau} \sum_{i=0}^{\text {ord } \tau} \tau^{i}(v)$. It turns out that the set ${ }^{\tau} R^{1}:=p(R)$ is again a root system called the folded root system. Its Weyl group is the fixed point Weyl group $\mathcal{W}^{\tau}:=\{w \in \mathcal{W}, \tau w=w \tau\}$. For irreducible $R$ the folded root systems are given by the following table:

\begin{tabular}{c|c|c|c|c|c}
$R$ & $\mathrm{~A}_{2 n-1}$ & $\mathrm{~A}_{2 n}$ & $\mathrm{D}_{n+1}, \tau^{2}=1$ & $\mathrm{E}_{6}$ & $\mathrm{D}_{4}, \tau^{3}=1$ \\
\hline${ }^{\tau} R^{1}$ & $\mathrm{C}_{n}$ & $\mathrm{BC}_{n}$ & $\mathrm{~B}_{n}$ & $\mathrm{~F}_{4}$ & $\mathrm{G}_{2}$
\end{tabular}.

Now consider a semisimple algebraic group $G$ over $k$ of type $R$. Here, $k$ is an algebraically closed field whose characteristic does not divide 2 or the order of the automorphism $\tau$. Our next aim is to lift this automorphism to the group $G$ itself. This will be accomplished as follows. 
First, fix a Borel subgroup $B<G$ and a maximal torus $T, B$. We write $\chi(T)$ for its character lattice. It is known that a lift of $\tau$ exists if and only if $\chi(T)$ is $\tau$-stable. Observe that the $\tau$ action on $\chi(T)$ fixes the action on $T$ completely. Secondly, choose a Chevalley basis for the Lie algebra Lie $G$, whose elements we denote by $h_{i} \in \operatorname{Lie} T, i \in$ $\{1, \ldots, \mathrm{rk} G\}, x_{\alpha} \in \operatorname{Lie} G_{\alpha}$. Then, we define the additive $\operatorname{root}$ groups $X_{\alpha}: k \rightarrow G, \alpha \in R$ via the exponential map $X_{\alpha}(c):=\exp \left(c x_{\alpha}\right)$. Now $\tau$ lifts uniquely to $G$ by imposing the condition $\tau\left(X_{\alpha}(c)\right)=X_{\tau(\alpha)}(c)$ for all simple roots $\alpha$ according to our choice of $B$ and $T$. But we can even describe the $\tau$ action on $G$ in a more detailed manner. The proof will be omitted because the result is rather standard in the theory of Chevalley groups, outlined, e.g., in [23]. A detailed proof of the second part can also be found in [15].

Proposition 2.1. (i) The automorphism $\tau$ lifts to $G$ if and only if the lattice $\chi(T)$ is $\tau$-stable. (This is automatically true for simple $G$ if $G$ is not of type $\mathrm{D}_{2 n}$.)

(ii) For a suitable parametrization of the root groups $X_{\alpha}$, the lift of $\tau$ acts on $G$ by the following rule:

(a) $\tau\left(X_{\alpha}(t)\right)=X_{\tau(\alpha)}(t) \forall t \in k, \alpha \in R$ if $G$ is simple not of type $\mathrm{A}_{2 n}$ or if $G$ is semisimple and $\tau$ a permutation of isomorphic factors of $G$.

(b) $\tau\left(X_{\alpha}(t)\right)=X_{\tau(\alpha)}\left((-1)^{\mathrm{ht} \alpha+1} t\right) \forall t \in k, \alpha \in R$ in case $G$ is of type $\mathrm{A}_{2 n}$.

By abuse of notation we will use the same symbol for the diagram automorphism and its lift to $G$. From [4, Section 13.3] and [24, Theorem 8.2] we get a description of the fixed point group $G^{\tau}$ and its identity component $G_{0}^{\tau}$.

Proposition 2.2. (i) The group $G_{0}^{\tau}$ is semisimple and $G^{\tau} / G_{0}^{\tau}$ is finite abelian. Additionally, for simply connected $G$ the group $G^{\tau}$ is connected.

(ii) For $G$ simple, the type of the group $G_{0}^{\tau}$ is given by the table:

\begin{tabular}{c|c|c|c|c|c} 
Type $G$ & $\mathrm{~A}_{2 n-1}$ & $\mathrm{~A}_{2 n}$ & $\mathrm{D}_{n+1}, \tau^{2}=1$ & $\mathrm{E}_{6}$ & $\mathrm{D}_{4}, \tau^{3}=1$ \\
\hline Type $G_{0}^{\tau}$ & $\mathrm{C}_{n}$ & $\mathrm{~B}_{n}$ & $\mathrm{~B}_{n}$ & $\mathrm{~F}_{4}$ & $\mathrm{G}_{2}$
\end{tabular}.

Now we can define the main object of our study, the non-connected group $\widetilde{G}$. Let $G$ be semisimple and $\Gamma \subset$ Aut $\Delta$ a subgroup of diagram automorphisms lifting to $G$. Then we set $\widetilde{G}:=G \rtimes \Gamma$. In this paper we will study the conjugacy action of $G$ on the $G$-coset $G \tau$ of $\widetilde{G}$. Now, following the same line of reasoning as in [21, Chapter I, §5], we can show that for simple $G$ and $x \in G \tau$, the conjugation map $G \rightarrow G$. $x$ is separable if $\operatorname{char}(k)$ is either zero or very good and does not divide the order of $\tau$. For details see [15, Section 1.4].

For the description of the representation theory of $\widetilde{G}$ we need another root system $R^{\prime}$ which is also embedded in $V^{\tau}$. It is constructed as follows. In all cases we set $R^{\prime}:=\left\{\alpha^{\prime}, \alpha \in R\right\}$.

(i) For $R$ irreducible not of type $\mathrm{A}_{2 n}$ we set $\alpha^{\prime}=\alpha$ if $\alpha=\tau(\alpha)$ and $\alpha^{\prime}=\sum_{i=1}^{\text {ord } \tau} \tau^{i}(\alpha)$ if $\alpha \neq \tau(\alpha)$.

(ii) For $R$ of type $A_{2 n}$ we define: $\alpha^{\prime}=(\alpha+\tau(\alpha))$ if $\alpha \neq \tau(\alpha)$ fulfilling $\check{\alpha}(\tau(\alpha))=0$ and $\alpha^{\prime}=2(\alpha+\tau(\alpha))$ if $\alpha \neq \tau(\alpha)$ with $\check{\alpha}(\tau(\alpha)) \neq 0$. (Note that $\alpha=\tau(\alpha)$ implies the existence of a $\operatorname{root} \beta$ with $\alpha=\beta+\tau(\beta)$ in this case. Then set $\alpha^{\prime}=\beta^{\prime}$.)

(iii) Let $R$ be reducible, the union of $n$ copies of an irreducible root system $\widetilde{R}$ and $\tau$ a permutation of the irreducible parts. Denote by $\Gamma$ the subgroup of $\operatorname{Aut}(R)$ generated 
by $\tau$, and then define

$$
\alpha^{\prime}=\frac{1}{\mid \Gamma_{\alpha}} \sum_{i=1}^{\mathrm{ord} \tau} \tau^{i}(\alpha)
$$

If $R$ is irreducible, a simple calculation yields the table

\begin{tabular}{c|c|c|c|c|c}
$R$ & $\mathrm{~A}_{2 n-1}$ & $\mathrm{~A}_{2 n}$ & $\mathrm{D}_{n+1}, \tau^{2}=1$ & $\mathrm{E}_{6}$ & $\mathrm{D}_{4}, \tau^{3}=1$ \\
\hline$R^{\prime}$ & $\mathrm{B}_{n}$ & $\mathrm{C}_{n}$ & $\mathrm{C}_{n}$ & $\mathrm{~F}_{4}$ & $\mathrm{G}_{2}$
\end{tabular}.

Thus we see that $R^{\prime}$ is the dual of the root system of $G_{0}^{\tau}$ in these cases. On the other hand, if $\tau$ is a permutation of the irreducible parts of a reducible root system $R$, then $R^{\prime}$ is clearly isomorphic to the folded root system ${ }^{\tau} R^{1}$. The introduction of $R^{\prime}$ is justified by the following observation, which follows by an easy computation. Here we denote by $\Lambda(R)$ the weight lattice of $R$.

Lemma 2.3. (i) There is a $\mathcal{W}^{\tau}$-equivariant isomorphism between the fixed point weight lattice $\Lambda(R)^{\tau}$ of $R$ with respect to $\tau$ and $\Lambda\left(R^{\prime}\right)$, the weight lattice of $R^{\prime}$.

(ii) Furthermore, the above identification implies an inclusion of root lattices $\mathbb{Z}(R)^{\tau} \supset$ $\mathbb{Z}\left(R^{\prime}\right)$

\section{Conjugacy classes and Invariant Theory}

In this section we will describe the invariant theory of the adjoint $G$-action on an exterior component $G \tau$ of $\widetilde{G}$. An investigation of the fibre structure of the corresponding quotient map provides an understanding of the conjugacy classes.

First, we introduce the notion of a Cartan subgroup generalising the concept of a maximal torus to non-connected linear algebraic groups $\widetilde{G}$. This notion is borrowed from the theory of compact groups and was introduced by Segal, cf. [3, Chapter IV.4]. Furthermore, we summarise its basic properties. More details can be found in [15, Chapter 2]. For $k=\mathbb{C}$ and $G$ adjoint, Gantmakher [7], see also [8] and [17, Sections 3.83.10 of Chapter 3], already introduced the definition below and proved the propositions up to Proposition 3.8. In the same setting Lemmas 3.7, 3.9 and partially 3.12 were already proved in [17].

Definition 1. An algebraic subgroup $C<\widetilde{G}$ is called a Cartan subgroup if the following properties hold:

(i) $C$ is diagonalisable,

(ii) $C$ has finite index in $N_{\widetilde{G}}(C)$, its normaliser in $\widetilde{G}$,

(iii) the component group $C / C_{0}$ of $C$ is cyclic.

The finite group $\mathcal{W}(C):=N_{G}(C) / C_{0}$ is called the outer Weyl group of $(\widetilde{G}, C)$ and is denoted by $\mathcal{W}(C)$.

In general, the existence of Cartan subgroups is not at all clear. If the identity component $G$ is reductive, however, we have a positive answer.

Proposition 3.1. Let $\widetilde{G}$ be as above with $G$ reductive. Then every semisimple element of $\widetilde{G}$ is contained in a Cartan subgroup $C$. 
Proof. We prove the proposition only for semisimple $G$. The general case is only slightly more difficult. It can be found in [15].

Let $g \in \widetilde{G}$ be a semisimple element, $C_{G}(g)_{0}$ the identity component of its centraliser in $G$ and $S<C_{G}(g)_{0}$ a maximal torus thereof. Denote by $H$ the algebraic subgroup of $\widetilde{G}$ generated by $S$ and $g$. Then we easily see that $H$ is diagonalisable. Since $H_{0}$ has to be a torus and since $S<H_{0}<C_{G}(g)_{0}$, we conclude that $H_{0}=S$. Now the finite group $H / S$ is cyclic. Indeed, a generating element is given by $g S$. Thus, we are left to show that $H$ has finite index in its normaliser in $\widetilde{G}$.

Consider the inequality:

$$
\begin{aligned}
{\left[N_{\widetilde{G}}(H): H\right] } & =\left[N_{\widetilde{G}}(H): C_{\widetilde{G}}(H)\right]\left[C_{\widetilde{G}}(H): H\right] \\
& \leq\left[N_{\widetilde{G}}(H): C_{\widetilde{G}}(H)\right]\left[C_{\widetilde{G}}(H): H_{0}\right] .
\end{aligned}
$$

The first factor on the right-hand side is finite. Indeed, this is true for arbitrary diagonalisable subgroups of algebraic groups, see, e.g., [25, Corollary 2]. For proving the finiteness of the second we proceed as follows. Combining the two inequalities

$$
\begin{aligned}
& S<C_{\widetilde{G}}(H)_{0}<C_{\widetilde{G}}(g)_{0}, \\
& S<C_{\widetilde{G}}(H)_{0}<C_{\widetilde{G}}(S)_{0}
\end{aligned}
$$

gives $S<C_{\widetilde{G}}(H)_{0}<C_{C_{\widetilde{G}}(g)_{0}}(S)_{0}$. By [24, Theorem 7.5, Theorem 8.2, and Corollary 9.4], $C_{\widetilde{G}}(g)_{0}$ is a reductive group and therefore $S=C_{C_{\widetilde{G}}(g)_{0}}(S)$. Hence $S=C_{\widetilde{G}}(H)_{0}$ and the proposition follows.

By the proof of the above proposition we also get a formula for the construction of a Cartan subgroup containing a given semisimple element $g \in \widetilde{G}$. Take the group generated by $g$ and a maximal torus of its centraliser $C_{G}(g)$.

The following is a kind of converse to this statement:

Lemma 3.2. Let $C$ be a Cartan subgroup of $\widetilde{G}$ and $g \in C$ an element, such that $g C_{0}$ generates $C / C_{0}$. Then $C_{0}$ is a maximal torus in $C_{G}(g)_{0}$.

Proof. Observe that $g$ and $C_{0}$ generate $C$ as an algebraic group. Assume that the statement of the lemma is false. Then $C_{0}$ is strictly contained in a certain maximal torus of $C_{G}(g)_{0}$. It will centralise $C$. This is a contradiction to property (ii) in the definition of Cartan subgroups.

Proposition 3.3. Let $C$ be a Cartan subgroup in $\widetilde{G}$. Then $C_{0}$ is a regular torus in $G$.

Proof. Since $C_{0}$ is a torus in $G$ we know that $C_{G}\left(C_{0}\right)$ is a reductive group. Consider the decomposition $C_{G}\left(C_{0}\right)=Z \widehat{G}$ into its centre $Z$ and its semisimple part $\widehat{G}=$ $\left(C_{G}\left(C_{0}\right), C_{G}\left(C_{0}\right)\right)$ with finite intersection. Clearly $C_{0} \subset Z$. Our aim is to show that $\widehat{G}$ is trivial. Take a element $g$ of $C$ generating $C / C_{0}$. Since conjugation with $g$ fixes $C_{0}$ pointwise it also stabilises $C_{G}\left(C_{0}\right)$ and therefore $\widehat{G}$. By Lemma $3.2, C_{0}$ is a maximal torus of the reductive group $C_{G}(g)_{0}$. Hence we have $C_{0}=C_{G}(g)_{0} \cap C_{G}\left(C_{0}\right)=C_{C_{G}(g)}\left(C_{0}\right)$. Therefore $\widehat{G}$ has only finitely many fixed points under conjugation with $g$. Now applying $[24,10.12]$, we conclude that $\widehat{G}$ is solvable. Being also semisimple it has to be trivial. 
For the sequel we restrict to the semidirect product case $\widetilde{G}=G \rtimes \Gamma$ with $G$ semisimple and $\Gamma$ a subgroup of diagram automorphisms of $G$. Additionally, fix an exterior component $G \tau$ for $\tau \in \Gamma$.

Proposition 3.4. Let $z \in G \tau$ be semisimple element and $C$ a Cartan subgroup containing $z$. Assume $z C_{0}$ to be a generator of $C / C_{0}$. Then every semisimple element $h \in G \tau$ is G-conjugate to an element of $C_{0} z$.

Proof. By [24, Theorem 7.5], there exists a pair $T<B$ consisting of a maximal torus $T$ and a Borel subgroup $B$ of $G$, both $z$-stable. Furthermore, we can even assume (after possibly conjugating with an element in $G$ ) that $T=N_{G}\left(C_{0}\right)$. Then, we clearly have $C_{0}=T_{0}^{z}$, the identity component of the $z$-fixed points of $T$. Similarly, there is a pair $T^{\prime}<B^{\prime}$ of a maximal torus and a Borel subgroup stabilised by $h$. After conjugating $h$ with an element in $G$ we can assume that $T=T^{\prime}$ and $B=B^{\prime}$. Then, $h z^{-1}$ is clearly an element in $G$ stabilising $T$ and $B$. This forces $h z^{-1} \in T$. Now the following lemma implies the statement of the proposition.

Lemma 3.5. Keeping the notation as above we have: Every element of $T z$ is $T$-conjugate to $T_{0}^{z} z$. In particular, every element in $T z$ is semisimple.

Proof. Denote by $c_{z}: \widetilde{G} \rightarrow \widetilde{G}, c_{z}(g)=z g z^{-1}$ the conjugacy action with $z$ on $\widetilde{G}$. Then $c_{z}$ is a semisimple automorphism of $\widetilde{G}$. We have to show that

$$
\forall h \in T \quad \exists h^{\prime} \in T / T_{0}^{z}: \quad h^{\prime} h z h^{\prime-1} \in T_{0}^{z} z .
$$

This is equivalent to the following equation

$$
\forall h \in T / T_{0}^{z} \quad \exists h^{\prime} \in T / T_{0}^{z}: \quad h^{\prime} c_{z}\left(h^{\prime-1}\right)=h^{-1},
$$

where $c_{z}$ operates on $T / T_{0}^{z}$ in the obvious manner.

By property (ii) in the definition of a Cartan subgroup, $c_{z}$ can have only finitely many fixed points on $T / T_{0}^{z}$. Applying [24, Theorem 10.1] implies the surjectivity of the conjugation map $T / T_{0}^{z} \rightarrow T / T_{0}^{z}$ given by $h^{\prime} \mapsto h^{\prime} c_{z}\left(h^{\prime-1}\right)$.

The next proposition describes the number of $G$-conjugacy classes of Cartan subgroups.

Proposition 3.6. The map $\Pi:\{$ Cartan subgroups of $\widetilde{G}\} \rightarrow\{$ cyclic subgroups of $\Gamma\}$ given by $\Pi(C)=C /(C \cap G)$ induces a bijection on the $G$-conjugacy classes of the former to the latter. In particular, we get $C \cap G=C_{0}$.

Proof. The surjectivity is clear by the construction following Proposition 3.1. For the injectivity, consider two Cartan subgroups $C$ and $C^{\prime}$ with $\Pi(C)=\Pi\left(C^{\prime}\right)$. Let $z$ be a generator of $C / C_{0}$ and $T$ the maximal torus $C_{G}\left(C_{0}\right)$. Then we have $C_{0}=T_{0}^{\tau}$ and $\tau(G \cap C)$ is a generator of $\Pi(C)$. Let $z^{\prime} \in C^{\prime} \cap G \tau$ be a generator of $C^{\prime} / C_{0}^{\prime}$. (This is always possible since the preimage of a generator of a surjective homomorphism $\mathbb{Z} / l \mathbb{Z} \rightarrow \mathbb{Z} / m \mathbb{Z}$ always contains a generator of $\mathbb{Z} / l \mathbb{Z}$.) By Proposition 3.4 every element $z^{\prime} \in C^{\prime} \cap G \tau$ is $G$-conjugate to an element in $T_{0}^{\tau} \tau$. Thus we assume $z^{\prime} \in T_{0}^{z} \tau$ and observe that there exists an exponent $m$ such that $z^{m} \in C_{0}^{\prime} \cap T$. Now choose $z^{\prime}$ such that $z^{\prime m}$ is regular 
in $T$, in addition. Then we get $C_{G}\left(z^{\prime}\right)_{0} \subset C_{G}\left(z^{\prime m}\right)_{0}=T$ implying $C_{G}\left(z^{\prime}\right)_{0}=T_{0}^{\tau}$. Since $C_{0}^{\prime}$ is a maximal torus of $C_{G}\left(z^{\prime}\right)$ we must have $C_{0}^{\prime}=T_{0}^{z}$ and hence $C=C^{\prime}$.

The second part of the theorem follows from the first. Indeed, let $C$ be any Cartan subgroup having $\tau \in \Gamma$ as a generator of $\Pi(C)$. Then $C$ has to be conjugate to $C^{\prime}=$ $T_{0}^{\tau} \times\langle\tau\rangle$. But we have $C \cap G=T_{0}^{\tau}$.

Lemma 3.7. Take a component $C^{*}$ of Cartan subgroup $C$ being a generator of $C / C_{0}$. Then any two elements in $C^{*}$ are conjugate under $G$ iff they are under $N_{G}(C)$.

Proof. Take two elements $x, y=g x g^{-1} \in C^{*}$ which are conjugate under $g \in G$. Then we have $x \in C \cap g^{-1} C g$. The group $C$ is clearly abelian. By Lemma 3.2 the two tori $C_{0}$ and $g^{-1} C_{0} g$ are maximal tori of $C_{G}(x)_{0}$. Thus there is an element $h \in C_{G}(x)_{0}$ with $h g^{-1} C_{0} g h^{-1}=C_{0}$. Since $x C_{0}$ respectively $x h g^{-1} C_{0} g h^{-1}$ generates the component group of $C / C_{0}$ respectively $h g^{-1} C g h^{-1} / h g^{-1} C_{0} g h^{-1}$, we get $g h^{-1} \in N_{G}(C)$.

An important prerequisite for the understanding of the invariant theory is the following density result:

Proposition 3.8. Let $\tau \in \Gamma$ and $\widetilde{G}$ be as above. Then the set of semisimple elements in $G \tau$ denoted by $(G \tau)_{\text {s.s. }}$ is a dense subset of $G \tau$.

Proof. Let $C$ be a Cartan subgroup of $\widetilde{G}$, such that $\tau C_{0}$ is a generator of $C / C_{0}$. Because of Proposition 3.4 it suffices to show that $\bigcup_{g \in G} g C_{0} \tau g^{-1}=(G \tau)_{\text {s.s. }}$ contains an open subset of $G \tau$. This will be shown by investigating the conjugation map $\Phi$ :

$$
\Phi: G / C_{0} \times C_{0} \rightarrow G \tau, \quad\left(g C_{0}, h\right) \mapsto g h \tau g^{-1} .
$$

Note that the dimensions of the image and preimage spaces coincide. Since $\Phi$ is a morphism of algebraic varieties, its image im $\Phi$ contains an open subset of its closure $\overline{\mathrm{im} \Phi}$. We shall prove the statement of the proposition using the dimension formula for morphisms. Thus, we have to find a point in $\operatorname{im} \Phi$ whose preimage contains an irreducible component of dimension zero. For every $t \tau \in C_{0} \tau$ there is an isomorphism $\Phi^{-1}(t \tau) \cong\left\{g \in G / C_{0} \mid g^{-1} t \tau g \in C_{0} \tau\right\}$. Now, choose $t \tau \in C$ such that $(t \tau)^{\text {ord } \tau}=$ $t^{\text {ord } \tau} \in C_{0}$ is a regular element in the maximal $T:=C_{G}\left(C_{0}\right)$ of $G$. Then we clearly have $\left\{g \in G / C_{0} \mid g^{-1} t \tau g \in C_{0} \tau\right\} \subset\left\{g \in G / C_{0} \mid g^{-1} t^{\text {ord } \tau} g \in T \tau\right\}$. Using the fact that any two elements in a maximal torus are conjugate in $G$ exactly when they are under $\mathcal{W}$, this larger set is given by $\left\{g \in G / C_{0} \mid g^{-1} t^{\text {ord } \tau} g \in T\right\}=N_{G}(T) C_{G}\left(t^{\text {ord } \tau}\right) / C_{0}$. By the regularity assumption the equality $C_{G}\left(t^{\text {ord } \tau}\right)_{0}=T$ holds. Hence, any connected component of $N_{G}(T) C_{G}\left(t^{\text {ord } \tau}\right) / C_{0}$ is isomorphic to $T / C_{0}$ as a variety. Thus, the union of all irreducible components of $\left\{g \in G / C_{0} \mid g^{-1} t \tau g \in C_{0} \tau\right\}$ contained in $T / C_{0}$ is given by the fixed point set $\left(T / C_{0}\right)^{t \tau}$ which is finite.

For the remainder of this section, let $C$ be a Cartan subgroup having $C_{0} \tau$ as a generator of its group of components $C / C_{0}$. Then for an appropriate maximal torus $T$ of $G$, the identity component $C_{0}$ equals $T_{0}^{\tau}$, the identity component of the $\tau$-fixed points of $T$. Denote by $\widetilde{\mathcal{W}}$ the corresponding outer Weyl group $\mathcal{W}(C)$. Its structure turns out to be important for our discussion.

Lemma 3.9. The forgetful map $\varphi: n T_{0}^{\tau} \mapsto n T$ for an element $n \in N_{G}(C)$, gives rise to a split exact sequence

$$
1 \longrightarrow\left(T / T_{0}^{\tau}\right)^{\tau} \longrightarrow \widetilde{\mathcal{W}} \stackrel{\varphi}{\longrightarrow} \mathcal{W}^{\tau} \longrightarrow 1
$$


Proof. Note that we have $\left(T / T_{0}^{\tau}\right)^{\tau} \subset \widetilde{\mathcal{W}}$.

First, we show $\operatorname{im} \varphi=\mathcal{W}^{\tau}$. Choose $n T_{0}^{\tau} \in \widetilde{\mathcal{W}}$. Then we have $\tau n \tau^{-1} \in n T_{0}^{\tau} \subset n T$, yielding $\operatorname{im} \varphi \subset \mathcal{W}^{\tau}$.

For the other inclusion, take $w=n T \in \mathcal{W}^{\tau}$. We have to find an element $t \in T$ such that $n t \in N_{G}(C)$. By assumption on $n$ there is a certain $t^{\prime} \in T$ such that $\tau^{-1} n \tau=n t^{\prime}$. We can even assume $t^{\prime} \in T_{0}^{\tau}$. Indeed, by Lemma 3.5 there is an element $\hat{t} \in T$, such that $\widehat{t} \tau\left(\widehat{t}^{-1}\right) t^{\prime} \in T_{0}^{\tau}$. Then we write $n$ for $n \widehat{t}$. Now a simple calculation shows that $n$ stabilises $C_{0}$ as well as $C_{0} \tau$ and thus $C$.

Second, exactness of the above sequence is proved: For $n T_{0}^{\tau} \in \operatorname{ker} \varphi$ we get $n \in T$. Additionally $n$ fulfils $n \tau n^{-1} \in \tau T_{0}^{\tau}$ implying $n T_{0}^{\tau}=\tau n T_{0}^{\tau} \tau^{-1}$.

Third, we show, that the sequence is split. Since $\mathcal{W}^{\tau}$ is the Weyl-group of the fixed point group $G_{0}^{\tau}$, and since $T_{0}^{\tau}$ is a maximal torus thereof we get an embedding $\iota: \mathcal{W}^{\tau} \hookrightarrow \widetilde{\mathcal{W}}$.

Let $\chi(T)$ be the character lattice of the maximal torus $T$ of $G$. Since the fixed point set $\chi(T)^{\tau}$ is again a lattice, it has to be a character lattice of a torus $T^{\prime}$, i.e., $\chi\left(T^{\prime}\right)=\chi(T)^{\tau}$. Then the inclusion $\chi(T)^{\tau} \subset \chi(T)$ corresponds to a projection $p: T \rightarrow T^{\prime}$. We get the interpretation of $T^{\prime}$ as the coinvariants of $T$ with respect to $\tau$.

Lemma 3.10. Let $\phi: T \rightarrow T$ be the group homomorphism $t \mapsto \phi(t)=t \tau t^{-1} \tau^{-1}$; then $T^{\prime} \cong T / \operatorname{im} \phi$. The map $\phi$ is $\mathcal{W}^{\tau}$-equivariant.

Proof. Denote by $\pi$ the projection map $T \rightarrow T / \operatorname{im} \phi$. For every character $\mu$ on the torus $T$ /im $\phi$, the element $\pi^{*}(\mu)$ is a character on $T$ being obviously $\tau$-invariant.

On the other hand take $\mu \in \chi(T)^{\tau}$. We define a character $\widehat{\mu}$ on $T / \operatorname{im} \phi$ by $\widehat{\mu}(t \operatorname{im} \phi):=$ $\mu(t)$. Then $\widehat{\mu}$ is well defined due to the $\tau$-invariance of $\mu$. Therefore, the character lattices of $T / \operatorname{im} \phi$ and $T^{\prime}$ coincide (as sublattices of $\chi(T)$ ). The $\mathcal{W}^{\tau}$-equivariance is trivial.

Now let us identify $T_{0}^{\tau}$ and $T_{0}^{\tau} \tau$ by right multiplication with $\tau$. This allows us to transport the $\widetilde{\mathcal{W}}$-action from $T_{0}^{\tau} \tau$ to $T_{0}^{\tau}$. This (twisted) action will be denoted by $*$. For $n_{w} \in N_{G}(C)$, a representative of $w \in \widetilde{\mathcal{W}}$, and $t \in T_{0}^{\tau}$ we set $w * t:=$ $n_{w} t \tau n_{w}^{-1} \tau^{-1}$. By construction, the restriction of the $*$-action to $\mathcal{W}^{\tau}$ is its usual action on $T_{0}^{\tau}$. Furthermore, consider the homomorphism $\left(T / T_{0}^{\tau}\right)^{\tau} \rightarrow T_{0}^{\tau}$ defined by $t T_{0}^{\tau} \mapsto$ $t \tau t^{-1} \tau^{-1}=t T_{0}^{\tau} * e$. Its image is a finite group denoted by $H$.

Lemma 3.11. Consider the commutative diagram with $\psi:=p \circ i$,

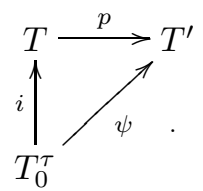

Then $\psi: T_{0}^{\tau} \rightarrow T^{\prime}$ is the quotient map of $T_{0}^{\tau}$ by the above defined $*$-action of $\left(T / T_{0}^{\tau}\right)^{\tau}$. It is $\mathcal{W}^{\tau}$-equivariant.

Proof. By the previous lemma, $T^{\prime}$ can be identified with the coinvariant torus of $T$. Now the surjectivity of $\psi$ is a direct consequence of Lemma 3.5. Furthermore, we clearly get $H \subset \operatorname{ker} \psi$. Now, let $\phi$ be the map defined in the previous lemma. Take an element $t \in \operatorname{ker} \psi=T_{0}^{\tau} \cap \operatorname{im} \phi$. Then we can find an element $s \in T$ with $t=s \tau s^{-1} \tau^{-1}$. We 
conclude $s \in\left(T / T_{0}^{\tau}\right)^{\tau}$. The $\mathcal{W}^{\tau}$-equivariance of $\psi$ follows from the $\mathcal{W}^{\tau}$-equivariance of $\phi$.

For simple and simply-connected $G$ the structure of $\left(T / T_{0}^{\tau}\right)^{\tau}$ can easily be described.

Lemma 3.12. Let $G$ be simply connected or adjoint and let $\tau$ be a diagram automorphism of $G$. Then the finite abelian group $\left(T / T_{0}^{\tau}\right)^{\tau}$ looks as follows: Let $n_{1}, \ldots, n_{s}$ be the orbit lengths of the $\Gamma=\langle\tau\rangle$-action on the set of simple roots. Then $\left(T / T_{0}^{\tau}\right)^{\tau}=$ $\mathbb{Z} / n_{1} \mathbb{Z} \times \ldots \times \mathbb{Z} / n_{s} \mathbb{Z}$. Furthermore, $T_{0}^{\tau}=T^{\tau}$ and $H \cong\left(T / T_{0}^{\tau}\right)^{\tau}$.

Proof. Since $G$ is either adjoint or simply connected its maximal tours is a direct product of the images of the fundamental co-weights, respectively the simple co-roots.

First, let us prove the connectedness statement and the first part of the lemma. Note that $\tau$ acts on the set of fundamental co-weights and the set of simple co-roots by permutation. Therefore we can assume that $\tau$ acts as a cyclic permutation on $T=\left(k^{*}\right)^{r}$,

$$
\tau\left(c_{1}, \ldots, c_{r}\right)=\left(c_{r}, c_{1}, \ldots, c_{r-1}\right)
$$

Then we immediately get $T_{0}^{\tau}=T^{\tau}=k^{*}(1, \ldots, 1)$ and $\left(T / T_{0}^{\tau}\right)^{\tau}$ is generated by the element $\left(1, \xi, \ldots, \xi^{r-1}\right) T_{0}^{\tau}$ where $\xi$ is a primitive $r$-th root of unity.

Now using $\phi$ as defined in Lemma 3.10 we see that $H=\left(\left(T / T_{0}^{\tau}\right)^{\tau}\right) /\left(T^{\tau} / T_{0}^{\tau}\right)$, whence the lemma.

Remark 2. (i) From the proof above we directly see that we always obtain $\left(T / T^{\tau}\right)^{\tau}=$ $(\mathbb{Z} / 2 \mathbb{Z})^{\operatorname{dim} T-\operatorname{dim} T_{0}^{\tau}}$ for $\tau$ of order 2 .

(ii) In the remaining simple cases, $G$ being $\operatorname{Spin}_{8}(k)$ or $\operatorname{PSO}_{8}(k)$ and $\tau$ of order 3, we obtain $\left(T / T^{\tau}\right)^{\tau} \cong \mathbb{Z} / 3 \mathbb{Z}$.

(iii) On the other hand, if $G$ is no longer simply connected, the lemma does not hold any longer, e.g., in the $S O_{2 n}$-case the group $\left(T / T_{0}^{\tau}\right)^{\tau}$ is isomorphic to $\mathbb{Z} / 2 \mathbb{Z}$ while $H$ is trivial. But it is still true that the cardinality of $H$ is a multiple of the order of $\tau$, cf. [15, Corollary 2.4].

Another key tool for the proof of the main result is to achieve an understanding of the representation theory of $\widetilde{G}$. In particular, the characters of the $\tau$-stable Weyl modules ${ }^{1}$ of $G$ turn out to provide a basis of the invariant $\operatorname{ring} k[G \tau]^{G}$.

Now the following lemma on invariant functions holds. The proof is rather straightforward and will therefore be omitted. It can be found in [15].

Lemma 3.13. Let $f \in k[G \tau]^{G}$ be an invariant function, $g \in G \tau$ and $g_{s}$ the semisimple part of $g$. Then $f(g)=f\left(g_{s}\right)$.

From now on we restrict to the case of cyclic $\Gamma$ generated by $\tau$. Following the methods of Mackey [14], the representation theory of $\widetilde{G}$ reduces to that of $G$ by a simple induction procedure. In the following $V(\lambda)$ denotes any finite-dimensional indecomposable $G$ module with highest weight $\lambda$ and highest weight vector $v_{\lambda}$.

Proposition 3.14. Let $G$ be simple, $\lambda \in \chi(T)$ a dominant character and $\Gamma_{\lambda}$ its stabiliser in $\Gamma$. Set $b:=\left|\Gamma_{\lambda}\right|$. Furthermore choose a primitive $b$-th root of unity $\xi$.

\footnotetext{
${ }^{1}$ We thank the referee for pointing out the use of Weyl modules instead of the irreducible ones in case of positive characteristic.
} 
(i) The G-module $\bigoplus_{i=1}^{\text {ord } \tau / b} V\left(\tau^{i}(\lambda)\right)$ affords b inequivalent indecomposable $\widetilde{G}$-representations. They will be denoted by $\widetilde{\rho}_{1}^{\lambda}, \ldots, \widetilde{\rho}_{\xi^{b-1}}^{\lambda}$. Here the index $\xi^{j}$ is the factor by which $\tau^{\text {ord } \tau / b}$ acts on the highest weight vector $v_{\lambda} \in V(\lambda)$.

(ii) If $\operatorname{char}(k)=0$, every irreducible representation of $\widetilde{G}$ is equivalent to one of them.

From now on the $V(\lambda)$ denotes the Weyl-module of $G$ with highest weight $\lambda$. If $\operatorname{char}(k)=0$ the Weyl modules are exactly the irreducible ones. Our next aim is to determine the corresponding characters. To do so we need some further notation. Let $R^{\prime}$ be the root system introduced in Section 2. Since we know that $\mathbb{Z}\left(R^{\prime}\right) \subset \chi(T)^{\tau}=$ $\chi\left(T^{\prime}\right) \subset \Lambda\left(R^{\prime}\right)$ there is a uniquely determined group $G^{\prime}$ of type $R^{\prime}$ having fundamental group $\Lambda\left(R^{\prime}\right) / \chi(T)^{\tau}$. We can thus identify $T^{\prime}$ with a maximal torus of $G^{\prime}$. We will write $V^{\prime}(\lambda)$ for its Weyl module of highest weight $\lambda$. Now denote by $X^{\lambda}$ the character of the Weyl module of the $G$-module with highest weight $\lambda$ and by $X^{\prime \lambda}$ that of $G^{\prime}$. Furthermore, we write $\widetilde{X}_{c}^{\lambda}$ for the character of the $\widetilde{G}$-representation $\widetilde{\rho}_{c}^{\lambda}$.

Proposition 3.15. The characters $\widetilde{X}_{c}^{\lambda}$ have the following shape.

(i) If $\tau(\lambda)=\lambda$, we have

$$
\begin{aligned}
\left.\tilde{X}_{c}^{\lambda}\right|_{G} & =X^{\lambda}, \\
\left.\tilde{X}_{c}^{\lambda}\right|_{G \tau} & \equiv c X^{\prime \lambda} .
\end{aligned}
$$

Here the denoted equivalence $\equiv$ is defined by considering the characters as elements in the group rings $\mathbb{Z}\left[\chi(T)^{\tau}\right]=\mathbb{Z}\left[\chi\left(T^{\prime}\right)\right]$.

(ii) If $\tau(\lambda) \neq \lambda$, we get with $b$ as in Proposition 3.14 :

$$
\begin{aligned}
\left.\tilde{X}_{c}^{\lambda}\right|_{G} & =\sum_{i=1}^{\mathrm{ord} \tau / b} X^{\tau^{i}(\lambda)}, \\
\left.\tilde{X}_{c}^{\lambda}\right|_{G \tau} & =0 .
\end{aligned}
$$

Proof. Recall that the representations $\widetilde{\rho}_{c}^{\lambda}$ are defined on the direct sum $\bigoplus_{i=1}^{\text {ord } \tau / b} V\left(\tau^{i}(\lambda)\right)$ by induction. Then $\left.\widetilde{X}_{c}^{\lambda}\right|_{G}$ clearly has the indicated shape in both cases. For $b<$ ord $\tau$, a representation matrix of $g \tau \in G \tau$ has block diagonal shape with only zeros on the diagonal with respect to the above direct sum decomposition, proving the second part of (ii).

Thus, we are left with proving the second statement of case (i): Clearly, we can assume $c=1$. Using the fact that $\left.\widetilde{X}_{c}^{\lambda}\right|_{G \tau}$ is an invariant function, Lemma 3.13 and Proposition 3.14 it is completely determined by its values on $T_{0}^{\tau} \tau$. Now consider the weight space-decomposition $V(\lambda)=\bigoplus_{\mu \in \chi(T)} V(\lambda)_{\mu}$. Every element $t \tau \in T_{0}^{\tau} \tau$ clearly interchanges the weight spaces $V(\lambda)_{\mu}$ and $V(\lambda)_{\tau(\mu)}$. Therefore, only the weight space $V(\lambda)_{\mu}$ with invariant weight $\mu=\tau(\mu)$ contributes to the trace,

$$
\tilde{X}^{\lambda}(t \tau)=\operatorname{tr} \widetilde{\rho}_{1}^{\lambda}(t \tau)=\left.\sum_{\mu \in \chi(T)^{\tau}} \mu(t) \operatorname{tr} \widetilde{\rho}_{1}^{\lambda}(\tau)\right|_{V(\lambda)_{\mu}} .
$$

The traces $\left.\operatorname{tr} \widetilde{\rho}_{1}^{\lambda}(\tau)\right|_{V(\lambda)_{\mu}}$ have already been calculated by Jantzen in $[9$, Chapter 9, Theorem 9]:

$$
\left.\operatorname{tr} \widetilde{\rho}_{1}^{\lambda}(\tau)\right|_{V(\lambda)_{\mu}}=\operatorname{dim} V^{\prime}(\lambda)_{\mu} .
$$

Since $\lambda, \mu \in \chi(T)^{\tau}=\chi\left(T^{\prime}\right)$ the expression on the right-hand side makes sense. Therefore we get

$$
\left.\widetilde{X}_{1}^{\lambda}\right|_{T_{0}^{\tau} \tau}=\sum_{\mu \in \chi(T)^{\tau}}\left(\operatorname{dim} V^{\prime}(\lambda)_{\mu}\right) \mu=\left.X^{\prime \lambda}\right|_{T^{\prime}} .
$$


With all these tools we are able to prove the main result.

Theorem 3.16. (i) The inclusion map $i: T_{0}^{\tau} \tau \rightarrow G \tau$ gives rise to a commutative diagram

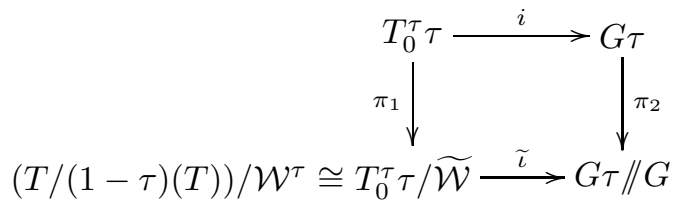

and an isomorphism $\widetilde{\iota}$.

(ii) Furthermore, $G \tau / / G \cong G \tau / / \widetilde{G}$.

Proof. (i) We have to show that the restriction map $i^{*}: k[G \tau] \rightarrow k\left[T_{0}^{\tau} \tau\right]$ induces an isomorphism on the invariant subrings $k[G \tau]^{G} \rightarrow k\left[T_{0}^{\tau} \tau\right]^{\mathcal{W}}$. Clearly, the inclusion $i^{*}\left(k[G \tau]^{G}\right) \subset k\left[T_{0}^{\tau} \tau\right]^{\widetilde{\mathcal{W}}}$ holds. The injectivity of $i^{*}$ is an immediate consequence of Propositions 3.4 and 3.8 .

The following claim implies that $i^{*}$ is surjective.

Claim. The set $i^{*}\left(\left\{\left.\widetilde{X}_{1}^{\lambda}\right|_{G \tau} \mid \lambda \in \chi(T)^{\tau}\right.\right.$ dominant $\left.\}\right)$ forms a basis of the invariant ring $k\left[T_{0}^{\tau} \tau\right]^{\widetilde{\mathcal{W}}}$.

Proof of Claim. Using the $\widetilde{\mathcal{W}}$-equivariant identification of $T_{0}^{\tau}$ and $T_{0}^{\tau} \tau$ as described in the paragraph preceding Lemma 3.11 and by Lemma 3.11 itself we have a sequence of rings

$$
k[G \tau]^{G} \hookrightarrow k\left[T_{0}^{\tau} \tau\right]^{\widetilde{\mathcal{W}}} \stackrel{\sim}{\longrightarrow}\left[T_{0}^{\tau} \tau /\left(T / T_{0}^{\tau}\right)^{\tau}\right]^{\mathcal{W}^{\tau}} \cong k\left[T^{\prime}\right]^{\mathcal{W}^{\tau}} .
$$

Write $\beta$ for the composition of the two maps in this equation. Now Proposition 3.15 implies $\beta\left(\left.\widetilde{X}_{1}^{\lambda}\right|_{G \tau}\right)=\left.X^{\prime \lambda}\right|_{T^{\prime}}$ for every character $\widetilde{X}_{1}^{\lambda}$ of $\widetilde{G}$ with invariant highest weight $\lambda=\tau(\lambda)$. Thus, we get

$$
\beta\left(\left\{\left.\widetilde{X}_{1}^{\lambda}\right|_{G \tau} \mid \lambda \in \chi(T)^{\tau} \text { dominant }\right\}\right)=\left\{\left.X_{1}^{\prime \prime}\right|_{T^{\prime}} \mid \lambda \in \chi\left(T^{\prime}\right) \text { dominant }\right\} .
$$

The set on the right-hand side forms a basis of $k\left[T^{\prime}\right]^{\mathcal{W}^{\tau}}$, cf. [22, Lemma 6.3], respectively $[25$, Section 3.4, Theorem 2].

(ii) Since the set of characters $\left\{\left.\widetilde{X}_{1}^{\lambda}\right|_{G \tau} \mid \lambda \in \chi(T)^{\tau}\right.$ dominant $\}$ generates the invariant ring $k[G \tau]^{G}$, this part follows immediately.

Remark 3. In characteristic 0 we can give the following much shorter proof of the Theorem. ${ }^{2}$ Using Proposition 3.8 and Lemma 3.7 the inclusion $i$ induces a bijective morphism $\widetilde{\iota}$ of the two quotient spaces which are clearly normal varieties. Using a result due to Richardson, see, e.g., [12, Section II.3.4., Lemma], $\widetilde{\iota}$ has to be an isomorphism. However, we believe that our proof provides us with some additional information: The quotient map can be described by characters and the quotient space is an affine space (under certain restrictions on the fundamental group of $G$ ).

As a consequence of the proof of the theorem above and [25, Section 3.4, Theorem 2], the quotient space is an affine space in the following cases.

\footnotetext{
${ }^{2}$ The author would like to thank E. B. Vinberg for pointing out this proof.
} 
Corollary 3.17. If the group $G^{\prime}$ is simply connected, i.e., $\chi(T)^{\tau}=\Lambda\left(R^{\prime}\right)$, the quotient space will be an affine space: $G \tau / / G \cong \mathbb{A}^{\mathrm{rk} G^{\prime}}$. Furthermore, the $\left.\widetilde{X}_{1}^{\lambda_{j}^{\prime}}\right|_{G \tau}, j \in\{1, \ldots, s\}$ freely generate $k[G \tau]^{G}$ as a $k$ algebra.

In particular, this happens in the following situations:

(a) If $G$ is of type $\mathrm{A}_{2 n}, \mathrm{E}_{6}$ or $\mathrm{D}_{4}$ with $\tau$ of order three.

(b) If $G$ is simply connected of type $\mathrm{A}_{2 n-1}$ or $\mathrm{D}_{n}$ with $\tau$ of order two.

Remark 4. Using the fact that the quotient is an affine space for simply connected $G$ allows us to determine explicitly the quotient spaces for arbitrary $G$. One must simply calculate the action on the quotient space of the kernel of the covering map $\widehat{G} \rightarrow G$. Here $\widehat{G}$ is the universal cover of $G$. Since we will not outline this here we refer to [15].

Making direct use of the Jordan decomposition we can, as in [18, Theorem 3.10], easily determine the fibre structure of the quotient map $\pi: G \tau \rightarrow T_{0}^{\tau} \tau / \widetilde{\mathcal{W}}$. We will skip the proof. It can be found in [15, Theorem 3.1]. For every element $t \tau \in T_{0}^{\tau} \tau$ denote by $C_{G}(t \tau)$ its centraliser in $G$ and by $V(t \tau)$ the unipotent variety of $C_{G}(t \tau)$.

Proposition 3.18. Let $t \tau$ be an element in $T_{0}^{\tau} \tau$. Then the following statements hold:

(i) $C_{G}(t \tau)$ is a reductive group

(ii) The reduced fibre $\pi^{-1}(\pi(t \tau))_{\mathrm{red}}$ over $\pi(t \tau)$ in $T_{0}^{\tau} / \widetilde{\mathcal{W}}$ is $G$-isomorphic to the associated fibre bundle $G \times{ }^{C_{G}}(t \tau) V(t \tau)$.

Particularly, each fibre consists of only finitely many orbits and contains exactly one regular and on semisimple orbit. Indeed, this follows directly from Lusztig's result on the finiteness of the number of unipotent orbits [13]. The semisimple orbit is the unique closed orbit in each fibre.

Let us conclude this section by giving a description of regular semisimple elements. Our discussion is based on [15, Section 3.2] and the explicit form of the action of $\Gamma$ on the root groups in Proposition 2.1. For simplicity let us assume that $r$ does not contain a component of $A_{2 n}$. An element $t \tau \in T_{0}^{\tau} \tau$ is clearly regular semisimple iff $C_{G}(t \tau)_{0}=T_{0}^{\tau}$. To achieve this, $t \tau$ has to avoid kernels of certain "roots". Take an arbitrary root $\alpha \in R$ and denote its $\Gamma$-orbit by $\alpha=\alpha_{1}, \ldots, \alpha_{l}$. Now, it is easy to see that $t \tau$ has a nontrivial fixed point on the product $X_{\alpha_{1}}(k) \ldots X_{\alpha_{l}}(k)$ of the images of the root groups, iff $\alpha(t)^{l}=1$. In this case we even get a one-parameter family of fixed points. Using the definition of the root system $R^{\prime}$ and how it is contained in $\mathbb{Z}\left({ }^{\tau} R^{1}\right)$ as well as $\mathbb{Z}(R)$, we get the following statement:

Corollary 3.19. (i) An element $t \tau \in T_{0}^{\tau} \tau$ is regular if and only if $\alpha^{\prime}(t) \neq 1$ for all $\alpha^{\prime} \in R^{\prime}$. (ii) Similarly, an element $t \tau \in T \tau$ is regular if and only if $\alpha^{\prime}(t) \neq 1$ for all $\alpha^{\prime} \in R^{\prime}$.

\section{The Steinberg cross section}

Under certain restriction on the fundamental group of $G$ we are able to construct, similarly as in [22], a section $C$ to the quotient map $\pi: G \tau \rightarrow T_{0}^{\tau} \tau / \widetilde{\mathcal{W}}$ having some additional nice properties.

Let us restrict to those semisimple groups $G$ with $\chi(T)^{\tau}=\Lambda(R)^{\tau}$. Then we know from Theorem 3.16 that our quotient is an affine space. 
For the construction of this section, start by choosing one representative $\alpha_{i}$ for each $\tau$-orbit on the set of simple roots $\Pi$. After relabelling we can assume that the simple roots chosen in this manner are $\alpha_{1}, \ldots, \alpha_{s}$. (Recall that $s=\operatorname{dim} G \tau / / G$.) Then the weights $\lambda_{1}^{\prime}, \ldots, \lambda_{s}^{\prime}$ form a set of generators of the weight lattice $\Lambda(R)^{\tau}=\Lambda\left(R^{\prime}\right)$. Let $X_{\alpha_{i}}, i \in\{1, \ldots, s\}$ denote the corresponding root groups of $G$ and let $n_{\alpha_{i}} \in N_{G}(T)$ be a representative of the corresponding simple reflection $s_{\alpha_{i}}$. Now we define

$$
C: \mathbb{A}^{s} \rightarrow G \tau, \quad\left(c_{1}, \ldots, c_{s}\right) \mapsto \prod_{i=1}^{s} X_{\alpha_{i}}\left(c_{i}\right) n_{\alpha_{i}} \tau
$$

Note that $C(0, \ldots, 0)=n_{\alpha_{1}} \ldots n_{\alpha_{s}} \tau$ is a representative in $N_{\widetilde{G}}(T)$ of a twisted Coxeter element, examined in [20, Section 7]. It has similar properties as the Coxeter element, e.g., it is unique up to conjugation in the Weyl group and has no eigenvector of eigenvalue one on the vector space generated by the root system.

This map $C$ has the properties of a section to the quotient map:

Theorem 4.1. The map $C$ is a section to the quotient map of the exterior component $G \tau$. More precisely, the following properties hold:

(i) $\operatorname{im} C$ is closed in $G \tau$.

(ii) $\left.\pi\right|_{\operatorname{im} C}: \operatorname{im} C \rightarrow G \tau / / G$ is an isomorphism.

(iii) $\operatorname{im} C$ meets each fibre exactly in its unique regular orbit.

Proof. (i) Let $\left\{\beta_{1}, \ldots, \beta_{s}\right\}$ denote the set of positive roots made negative by the inverse of our twisted Coxeter element $\tau^{-1} s_{\alpha_{s}} \ldots s_{\alpha_{1}}$. Then we obtain

$$
\operatorname{im} C=\left(\prod_{i=1}^{s} \operatorname{im} X_{\beta_{i}}\right) n_{\alpha_{1}} \ldots n_{\alpha_{s}} \tau
$$

Hence im $C$ is a closed subset of $G \tau$.

(ii) For the proof of this part of the theorem we have to evaluate the quotient map on $\operatorname{im} C$. By Corollary 3.17, the quotient map $\pi$ has the form

$$
\pi(g \tau)=\left(\widetilde{X}_{1}^{\lambda_{1}^{\prime}}(g \tau), \ldots, \widetilde{X}_{1}^{\lambda_{s}^{\prime}}(g \tau)\right), \quad \forall g \in G .
$$

Now denote by $V\left(\lambda_{i}^{\prime}\right)$ the irreducible $G$-module with highest weight $\lambda_{i}^{\prime}$, the weight space of weight $\mu$ by $V\left(\lambda_{i}^{\prime}\right)_{\mu}$, its canonical inclusion $V\left(\lambda_{i}^{\prime}\right)_{\mu} \hookrightarrow V\left(\lambda_{i}^{\prime}\right)$ by $\iota_{\mu}$ and the canonical projection $V\left(\lambda_{i}^{\prime}\right) \rightarrow V\left(\lambda_{i}^{\prime}\right)_{\mu}$ by $\pi_{\mu}$. With this notation we clearly have

$$
\tilde{X}_{1}^{\lambda_{i}^{\prime}}(g \tau)=\sum_{\mu \in \chi(T)} \operatorname{tr}_{V\left(\lambda_{i}^{\prime}\right)_{\mu}}\left(\pi_{\mu} \widetilde{\rho}_{1}^{\lambda_{i}^{\prime}}(g \tau) \iota_{\mu}\right) .
$$

The following well-known lemma describes the action of $\tau$, the Weyl group and the root groups on the weight spaces.

Lemma 4.2. Let $G$ be a reductive group, $V$ a finite-dimensional $G$-module and $V_{\lambda}$ a weight space. Then we have for every $v \in V_{\lambda}$ :

(i) $X_{\alpha}(c) . v=\sum_{i=0}^{\infty} c^{i} v_{i}$, where $v_{i} \in V_{\lambda+i \alpha}$ is independent of $c$ and $v_{0}=v$.

Furthermore, the following holds:

(ii) $n_{\alpha} \cdot v \in V_{s_{\alpha}(\lambda)}=V_{\lambda-\check{\alpha}(\lambda) \alpha}$.

(iii) $\tau . v \in V_{\tau(\lambda)}$.

By the next result we only have to consider weights which are invariant under $\tau$. 
Lemma 4.3. For $g \tau \in \operatorname{im} C$ we have

$$
\operatorname{tr}_{V\left(\lambda_{i}^{\prime}\right)_{\mu}}\left(\pi_{\mu} \widetilde{\rho}_{1}^{\lambda_{i}^{\prime}}(g \tau) \iota_{\mu}\right)=0,
$$

if $\tau(\mu) \neq \mu$. Thus our characters take the following values:

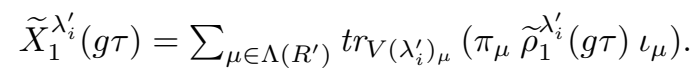

Proof. By Lemma 4.2 we have to show that $\tau(\mu) \neq \mu$ implies $\mu \notin \tau(\mu)+\mathbb{Z}\left(\alpha_{1}, \ldots, \alpha_{s}\right)$. Assuming the contrary we find $p_{j} \in \mathbb{Z}, j \in\{1, \ldots, s\}$ fulfilling the equation

$$
0 \neq \mu-\tau(\mu)=\sum_{j=1}^{s} p_{j} \alpha_{j}
$$

We always have the equality

$$
\sum_{i=1}^{\mathrm{ord} \tau} \tau^{i}(\mu-\tau(\mu))=0
$$

Combining the last two equations we get

$$
0=\sum_{\alpha \in \Pi} p_{\alpha}^{\prime} \alpha
$$

Here the $p_{\alpha}^{\prime}$ are equal to the $p_{i}$ up to a positive constant depending on $\alpha$. Hence $p_{\alpha}^{\prime}=p_{i}=0$, which is absurd.

Now a straightforward calculation yields the following equality for $\mu \in \Lambda(R)$ :

$$
\operatorname{tr}_{V\left(\lambda_{i}^{\prime}\right)_{\mu}}\left(\pi_{\mu}\left(\prod_{i=1}^{s} X_{\alpha_{i}}\left(c_{i}\right) n_{\alpha_{i}}\right) \tau \iota_{\mu}\right)=\operatorname{tr}_{V\left(\lambda_{i}^{\prime}\right)_{\mu}}\left(\prod_{i=1}^{s}\left(\pi_{\mu} X_{\alpha_{i}}\left(c_{i}\right) n_{\alpha_{i}} \iota_{\mu}\right) \pi_{\mu} \tau \iota_{\mu}\right) \text {. }
$$

Combining this equation with Lemma 4.2 we clearly get for $\mu=\sum_{j=1}^{s} m_{j} \lambda_{j}$ and certain $a_{\mu} \in k$,

$$
\operatorname{tr}_{V\left(\lambda_{i}^{\prime}\right)_{\mu}}\left(\pi_{\mu} C\left(c_{1}, \ldots, c_{s}\right) \iota_{\mu}\right)=\left\{\begin{array}{cl}
a_{\mu} c_{1}^{m_{1}} \ldots c_{s}^{m_{s}}, & \text { if } \mu \text { is dominant } \\
0, & \text { otherwise. }
\end{array}\right.
$$

Let us continue by introducing an order relation $\geq$ on the set of fundamental dominant weights of $\Lambda\left(R^{\prime}\right)$ according to the following rule:

$$
\begin{aligned}
& \lambda_{i}^{\prime}>\lambda_{j}^{\prime} \text { if there exists a dominant weight } \lambda^{\prime} \in \Lambda\left(R^{\prime}\right) \text {, such that } \lambda^{\prime} \prec \lambda_{i}^{\prime} \\
& \left(\text { in } \Lambda\left(R^{\prime}\right) !\right) \text {, i.e., } \lambda_{i}^{\prime}-\lambda^{\prime} \text { is a sum of positive roots and there exist } m_{k} \geq 0 \\
& \text { with } m_{j}>0 \text { fulfilling } \\
& \qquad \lambda^{\prime}=\sum_{k=1}^{s} m_{k} \lambda_{k}^{\prime} .
\end{aligned}
$$

Furthermore, $\lambda_{i}^{\prime} \geq \lambda_{j}^{\prime}$, if either $\lambda_{i}^{\prime} \dot{>} \lambda_{j}^{\prime}$ or $\lambda_{i}^{\prime}=\lambda_{j}^{\prime}$.

With these preparations we can evaluate the characters on the image of the section $C$, i.e., we obtain Equation 30, where $a_{\lambda_{i}^{\prime}} \in k$ and where $P_{i}$ is a polynomial in those $c_{j}$ with $\lambda_{j}^{\prime} \leq \lambda_{i}^{\prime}$ and $\lambda_{j}^{\prime} \neq \lambda_{i}^{\prime}$.

$$
\tilde{X}_{1}^{\lambda_{i}^{\prime}}\left(C\left(c_{1}, \ldots, c_{s}\right)\right)=a_{\lambda_{i}^{\prime}} c_{i}+P_{i}\left(c_{1}, \ldots, c_{s}\right) .
$$


Now the claim follows if we could show that $a_{\lambda_{i}^{\prime}} \neq 0$ : By the reasoning above the only contribution to the coefficient $a_{\lambda_{i}^{\prime}}$ of $c_{i}$ comes from the action of $C\left(c_{1}, \ldots, c_{s}\right)$ on the weight space corresponding to the highest weight. Let $v \in V\left(\lambda_{i}^{\prime}\right)$ be a highest weight vector. By definition we have $\tau \cdot v=v$. Furthermore, one easily calculates for $j \neq i$ :

$$
X_{\alpha_{j}}\left(c_{j}\right) n_{\alpha_{j}} \cdot v=v .
$$

Clearly, we are left to investigate $X_{\alpha_{i}}\left(c_{i}\right) n_{\alpha_{i}} \cdot v$. Consider the subgroup

$$
G_{\alpha_{i}}:=\left\langle\operatorname{im} X_{\alpha_{i}}, \operatorname{im} X_{-\alpha_{i}}\right\rangle
$$

of $G$. It is easily seen that it is isomorphic to $\mathrm{SL}_{2}(k)$ acting on $V\left(\lambda_{i}^{\prime}\right)_{\lambda_{i}^{\prime}} \oplus V\left(\lambda_{i}^{\prime}\right)_{\lambda_{i}^{\prime}-\alpha_{i}}$ in its natural representation. Using this identification we have $v=\left[\begin{array}{l}1 \\ 0\end{array}\right]$ and $X_{\alpha_{i}}\left(c_{i}\right)=\left[\begin{array}{cc}1 & c_{i} \\ 0 & 1\end{array}\right]$. By multiplying $n_{\alpha_{i}}$ with an element in $T$ we can assume $n_{\alpha_{i}}=\left[\begin{array}{rr}0 & 1 \\ -1 & 0\end{array}\right]$.

(iii) This part is an easy consequence of Theorem 4.4.

Theorem 4.4. Let $g \tau$ be in $G \tau$. Then the following three properties are equivalent:

(i) $g \tau$ is regular.

(ii) The derivative $(d \pi)_{g \tau}$ of $\pi$ in $g \tau$ is surjective.

(iii) $g \tau$ is $G$-conjugate to an element of im $C$.

Proof. (iii) $\Rightarrow$ (ii): Follows immediately from Theorem 4.1(ii).

(ii) $\Rightarrow(\mathrm{i})$ : This part will be proved by showing that the differentials $\left(\left.d \widetilde{X}_{1}^{\lambda_{i}^{\prime}}\right|_{G \tau}\right)_{g \tau}$, $i \in\{1, \ldots, s\}$ are linearly dependent at irregular $g \tau$. The proof proceeds in several steps along similar lines as in [22].

1. Observe that the set of semisimple irregular elements in $G \tau$ is dense in the set of irregular elements. This is proved along the lines of reasoning in [22, Section 5]; for details see [15, Theorem 4.1]. Since the set $\left\{g \tau \in G \tau \mid\left(\left.d \widetilde{X}_{1}^{\lambda_{i}^{\prime}}\right|_{G \tau}\right)_{g \tau}, i \in\right.$ $\{1, \ldots, s\}$ are linearly dependent $\}$ is a closed subset we may thus assume $g \tau$ to be irregular and semisimple. After conjugating we can even choose $g \tau=t \tau \in T \tau$ for a given $\tau$-stable maximal torus $T$.

2. Now let us fix a Borel subgroup $B \supset T$. Denote its unipotent radical by $U$ and the unipotent radical of the opposite Borel by $U^{-}$. Furthermore, let $\mathfrak{t}$ be the tangent space $T_{t \tau}(T \tau) \subset T_{t \tau}(G \tau)$. We claim:

For every $F \in k[G \tau]^{G}$ the following statement holds:

$$
(d F)_{t \tau}=0 \Longleftrightarrow\left(\left.d F\right|_{T \tau}\right)_{t \tau}=\left.(d F)_{t \tau}\right|_{\mathfrak{t}}=0 .
$$

Proof of claim. Consider the open 'Bruhat cell' $U^{-} T U \tau \subset G \tau$ and let $\Psi$ be the well-known isomorphism

$$
\begin{aligned}
\Psi: \mathbb{A}^{|R|} \times\left(k^{*}\right)^{r} & \rightarrow U^{-} T U \tau \\
\left(\left(u_{\alpha}\right)_{\alpha \in R^{+}},\left(v_{\alpha}\right)_{\alpha \in R^{+}}, t_{1}, \ldots, t_{r}\right) & \mapsto \prod_{\alpha \prec 0} X_{\alpha}\left(u_{-\alpha}\right) \prod_{i=1}^{r} \nu_{i}\left(t_{i}\right) \tau \prod_{\alpha \succ 0} X_{\alpha}\left(v_{\alpha}\right) .
\end{aligned}
$$

Here the $X_{\alpha}, \alpha \in R$, are the root groups and the $\nu_{i}, i \in\{1, \ldots, r\}$ are one-parameter multiplicative groups spanning $T$. Accordingly, every tangent vector $Y \in T_{t \tau}(G \tau)$ 
decomposes uniquely into a sum $Y=Y_{1}+Y_{2}$ with $Y_{1} \in T_{t \tau} U^{-} t U \tau$ and $Y_{2} \in T_{t \tau} T \tau$. Now $t^{\prime} \in T_{0}^{\tau}$ acts on $U^{-} T U \tau$ by conjugation:

$$
\begin{array}{r}
t^{\prime} .\left(\left(u_{\alpha}\right)_{\alpha \in R^{+}},\left(v_{\alpha}\right)_{\alpha \in R^{+}}, t_{1}, \ldots, t_{r}\right) \\
\quad=\left(\left(\alpha\left(t^{\prime}\right)^{-1} u_{\alpha}\right)_{\alpha \in R^{+}},\left(\alpha\left(t^{\prime}\right) v_{\alpha}\right)_{\alpha \in R^{+}}, t_{1}, \ldots, t_{r}\right) .
\end{array}
$$

The invariant function $F$ can uniquely be written as a sum $F=F_{1}+F_{2}$, where $\Psi^{*}\left(F_{1}\right)$ is a polynomial only in the powers of the $t_{i}^{ \pm 1}$, while at least one factor in the $u_{\alpha}$ or the $v_{\alpha}$ appears in each monomial of $\Psi^{*}\left(F_{2}\right)$. Since $F$ and $F_{1}$ are obviously $T_{0}^{\tau}$ invariant, $F_{2}$ has to be so as well. Recall that $T_{0}^{\tau}$ is a regular torus, cf. Proposition 3.3. Therefore, every monomial of $\Psi^{*}\left(F_{2}\right)$ must contain at least two factors from the set $\left\{u_{\alpha}, v_{\alpha}\right\}$. This implies $\left(d F_{2}\right)_{t \tau}(Y)=0$. Now the claim follows:

$$
d F_{t \tau}(Y)=\left(d F_{1}\right)_{t \tau}(Y)=\left(d F_{1}\right)_{t \tau}\left(Y_{2}\right)=d F_{t \tau}\left(Y_{2}\right)
$$

3. Consider the quotient map $p: T \tau \rightarrow T \tau / / T=T^{\prime}$ from Section 3. Recall that $T^{\prime}$ is a maximal torus of a semisimple algebraic group $G^{\prime}$ with root system $R^{\prime}$. By the description of irregular elements in $T \tau$, cf. Corollary 3.19, we see that $t \tau$ is regular in $T \tau$ if and only if $p(t \tau)$ is regular in $T^{\prime}$.

4. For every $T$-invariant function $F$ on $T \tau$ we denote by $\widehat{F}$ its corresponding function on the quotient space $T^{\prime}$. By Lemma 3.10 the differential $(d p)_{t \tau}$ is always surjective. Thus

$$
(d F)_{t \tau}=0 \Longleftrightarrow(d \widehat{F})_{p(t \tau)}=0 .
$$

5. By $\left[25\right.$, Section 3.8, Lemma p. 125] we know that $\left(\left.d X^{\prime \lambda_{i}^{\prime}}\right|_{T^{\prime}}\right)_{t^{\prime}}, i \in\{1, \ldots, s\}$ are linearly dependent for $t^{\prime} \in T^{\prime}$ for irregular $t^{\prime}$.

(i) $\Rightarrow$ (iii): Let $g \tau \in G \tau$ be regular. Take $h \tau \in \operatorname{im} C \cap \pi^{-1}(\pi(g \tau))$. Since we already know that $h \tau$ is regular the statement follows from Proposition 3.18.

Using a standard reasoning in algebraic geometry, as outlined in [25, Section 3.8, Theorem 7], we derive the following corollary. For a detailed proof see [15, Proposition 5.2].

Corollary 4.5. The quotient map $\pi: G \tau \rightarrow G \tau / / G$ is flat and its schematic fibres are reduced and normal.

\section{Complements}

Let us mention here some further results. First, we describe Grothendieck's simultaneous resolution.

Consider the associated bundle $G \times{ }^{B} B \tau$. The class of $(g, b)$ in $G \times{ }^{B} B \tau$ will be denoted by $g * b \tau$. Now define the conjugacy map $\Phi: G \times{ }^{B} B \tau \rightarrow G \tau$ by $g * b \tau \mapsto g b \tau g^{-1}$. Furthermore, denote by $\Theta$ the quotient map $G \times{ }^{B} B \tau \mapsto B \tau / / B \cong T \tau / / T=: T^{\prime}$ and by $\Psi$ the quotient of $T^{\prime}$ by $\mathcal{W}^{\tau}$. 
Theorem 5.1. Assume that $\tau$ acts without fixed points on the fundamental group of $G$. Furthermore, let $\operatorname{char}(k)>r k G+1$ or $\operatorname{char}(k)=0$. Then the following diagram gives a simultaneous resolution of $\pi: G \tau \rightarrow G \tau / / G$ :

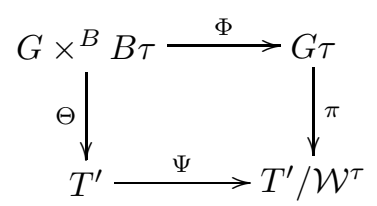

Here simultaneous resolution means that $\Psi$ is finite and surjective, $\Theta$ is smooth, $\Phi$ is proper and $\Phi$ restricted to the fibres of $\Theta$ provides a resolution of singularities of the fibres of $\pi$. Additionally the above diagram shall be commutative.

In Section 3 we proved that every semisimple element in $G \tau$ is $G$-conjugate to an element in $T_{0}^{\tau} \tau$. Therefore, a natural question to ask is whether every element of $G \tau$ is $G$-conjugate to an element in the shifted connected fixed point set $G_{0}^{\tau} \tau$. As we have shown in [15, Appendix], this does not hold for simple $G$ (as soon as $\tau$ is a non-trivial outer automorphism of $G$ ). This is due to the fact that the semisimple part of the centraliser in $G_{0}^{\tau}$ of a given element $t \tau \in T_{0}^{\tau} \tau$ may be strictly smaller than that in $G$.

Acknowledgements. Thanks are due to P. Slodowy not only for initiating this line of research but also for many helpful discussions. I also like to thank K. Saito for hospitality during my stays at RIMS in Kyoto. Financial support by the Schweizerischer Nationalfonds is gratefully acknowledged.

\section{References}

[1] A. Alekseev, V. Schomerus, D-branes in the WZW-model, Phys. Rev. D60 (1999), 0619011-061901-2.

[2] E. Brieskorn, Die Auflösung der rationalen Singularitäten holomorpher Abbildungen, Math. Ann. 178 (1968), 255-270.

[3] T. Bröcker, T. tom Dieck, Representations of Compact Lie Groups, Springer, 1985.

[4] R. W. Carter, Simple Groups of Lie Type, John Wiley \& Sons, 1972.

[5] F. Digne, J. Michel, Groupes réductifs non connexes, Ann. Sci. Éc. Borm. Sup. 27 (1994), 345-406.

[6] G. Felder, J. Fröhlich, J. Fuchs, C. Schweigert, The Geometry of WZW-branes, J. Geom. Phys. 34 (2000), 162-190.

[7] F. R. Gantmacher, Canonical representation of automorphisms of a complex semisimple Lie group, Мат. Сборник 5 (1939), No. 1, 101-144.

[8] N. Jacobson, A note on automorphisms of Lie algebras, Pac. J. Math. 12 (1962), 303-314.

[9] J. C. Jantzen, Darstellungen Halbeinfacher Algebraischer Gruppen, Bonner Math. Schriften, Bd. 67, 1973.

[10] D. Joyner, On finite dimensional representations of non-connected reductive groups, J. Lie Theory 10 (2000), 269-284.

[11] B. Kostant, Lie algebra cohomology and the generalized Borel-Weyl theorem, Ann. Math. 74 (1961), No. 2, 329-390. 
[12] H. Kraft, Geometrische Methoden in der Invaraintentheorie, 2nd Ed., Aspects Math., Vieweg, 1985. Russ transl.: X. Крафт, Геометрические методы в теории инвариантов, М., Мир, 1987.

[13] G. Lusztig, On the finiteness of the number of nilpotent classes, Invent. Math. 34 (1976), 201-213.

[14] G. Mackey, Unitary representations of group extensions, I, Acta Math. 99 (1958), 265-311.

[15] S. Mohrdieck, Conjugacy Classes of Non-Connected Semisimple Algebraic Groups, Ph.D thesis, Univ. Hamburg, 2000, www.sub.uni-hamburg.de/emedien/edissertationen.html.

[16] S. Mohrdieck, R. Wendt, Twisted conjugacy classes, coadjoint orbits of loop groups, and $D$-branes in the WZW-model, preprint, arXiv: QA0303118, 2003.

[17] Э. Б. Винберг, В. В. Горбацевич, А. Л. Онищик, Группи и алгебры Ли, ІІІ. Строение групп и алгебр Ли, Итоги науки и техн., Совр. пробл. матем., Фунд. направл., том 41, М., ВИНИТИ, 1990. Engl. transl.: А. L. Onishchik, Е. В. Vinberg, V. V. Gorbatsevich, Lie Groups and Lie Algebras, III. Structure of Lie Groups and Lie Algebras, Encycl. Math. Sci., Vol. 41, Springer, Berlin, 1994.

[18] P. Slodowy, Simple Singularities and Simple Algebraic Groups, Lect. Notes Math., Vol. 815, Springer, 1980

[19] N. Spaltenstein, Dégénérescences des formes bilinéaires, J. Algebra 80 (1983), 1-28.

[20] T. A. Springer, Regular elements of finite reflection groups, Invent. Math. 25 (1974), $159-198$

[21] T. A. Springer, R. Steinberg, Conjugacy classes in algebraic groups, in: Seminar on Algebraic Groups and Related Finite Groups, Lect. Notes Math., Vol. 131, Springer, 1970, pp. E-1-E-100.

[22] R. Steinberg, Regular elements of semisimple algebraic groups, Publ. Math. I.H.E.S. 25 (1965), 49-80.

[23] R. Steinberg, Lectures on Chevalley Groups, Lect. Notes, Yale University, 1967. Russ. transl.: Р. Стейнберг, Лекции о группах Шевалле, М., Мир, 1975.

[24] R. Steinberg, Endomorphisms of Linear Algebraic Groups, Memoires of the AMS, Vol. 80, 1968.

[25] R. Steinberg, Conjugacy Classes in Algebraic Groups, Lect. Notes Math., Vol. 366, Springer, 1974. 